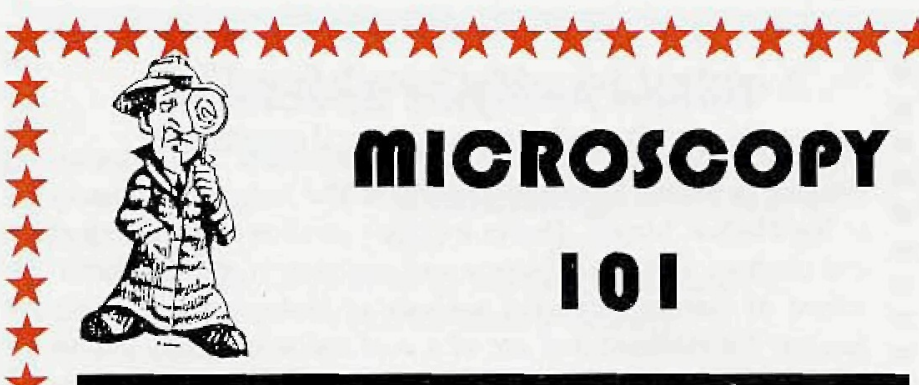

Just as we hope that you are enjoying this feature, edited by Phil Oshel, we hope that you will assist Phil by making your own contributions - which can be in any microscopy area and must only be interest to other working microscopists. Contributions and may be sent to Phil Oshel by:

eMail: $\quad$ oshel@ux1.cso.uiuc.edu

Snail Mail: Station A

PO Box 5037

Champaign IL 61825-5037

\section{TEM Contamination And Plasma Cleaning}

During the past three years I have concentrated a great deal of effort in addressing possible sources of TEM contamination and developing technology to reduce, if not eliminate this contamination from hydrocarbons.

We have discovered that the major source of hydrocarbon contamination is either the specimen or the specimen holder. Some of the possible causes are diffusion pumped or mechanical rotary backing pumped ion mills, failure to fully remove cleaning chemicals such as acetone and alcohol from the specimen, and adhesive residue on the specimen (popular adhesives for attaching specimens during dimpling/ tripod polishing can be hydrocarbon based). Additionally, inadvertent touching of the specimen or specimen holder in front of the vacuum sealing 0 -ring is also a major cause of contamination.

The technology that we have found to be extremely effective is a low-energy, high frequency oxygen/argon plasma. The plasma is created in a vacuum chamber which is designed to accept the specimen and the specimen holder. The specimen holder is exposed to the plasma from the o-ring forward. Pumping is achieved by an oil-free vacuum system in order to eliminate any possibility of backstreaming oil from the vacuum system into the plasma chamber. This is a critical aspect of the technology.

Ion energies are very low and the contamination is removed by means of a chemical reduction of hydrocarbons by the oxygen radicals created within the plasma.

We have found that the vacuum systems of the newer TEMs are actually quite clean. The best decontamination for high-quality microanalysis is to subject the specimen to plasma cleaning before putting the specimen or holder in the TEM. Cleaning times are on the order of one or two minutes for most specimens. This process results in maintaining both microscope vacuum cleanliness and specimen integrity. It has also been found that once a carbon spot has been created by the electron beam it is very difficult to remove.

The effectiveness of plasma cleaning is realized in TEMs with high-brightness guns (LaB6 and FEG). Contamination can also be a factor in TEMs with W filament sources, however, the high current density of the FEG often times can result in unacceptable levels of contamination when conducting fine-probe microanalysis (EDS or PEELS).

Cryotechnology and flooding of the specimen with the electron beam are acceptable techniques to work around the problem. It is far better to eliminate the problem at the source.

Paul E. Fischione, E.A. Fischione Instruments, Inc.

\section{Peak Overlaps in EDXWDX of Titanium and Vanadium}

The general case for interference with the transition elements is that the $\mathrm{Z}-1$ element $\mathrm{Kb}$ peak overlaps the $\mathrm{Z}$ element $\mathrm{Ka}$ peak. Ti $\mathrm{Kb}$ on $\mathrm{VKa}$ is a case in point

For EDS analysis, assuming that linear least squares deconvolution is used, the fit for Ti should handle both Ti Ka and $\mathrm{Kb}$ if they are grouped together as an EDS reference. Any residual could then appear as a positive value for $V$. This may reflect a calibration difference between the sample and reference spectra (I'm assuming we are not talking standardless EDS here).

For WDS measurement of Ti and $\mathrm{V}$, one has the choice of PET or LIF analyzing crystals; the latter has better resolution and should be used to reduce the magnitude of the overlap of $\mathrm{Ti} \mathrm{Kb}$ on $\mathrm{V} \mathrm{Ka}$ to begin with. I use $\mathrm{TiO}_{2}$ and $\mathrm{V}_{2} \mathrm{O}_{5}$ crystals, both synthetic and pure, as primary standards for $T i$ and $V$, respectively. The $\mathrm{TiO}_{2}$ is known to not contain any $\mathrm{V}$ - this is an important requirement for its use in interference correction. I find that (using LIF) the measured $\mathrm{V} \mathrm{Ka}$ k-ratio on my synthetic $\mathrm{TiO}_{2}$ is typically less than 0.0050 (i.e. approximately $0.5 \%$ as $\mathrm{V}_{2} \mathrm{O}_{5}$ ). This measured $\mathrm{V} \mathrm{K}$-ratio on $\mathrm{TiO}_{2}$ is really just due to the intensity of $T i \mathrm{~Kb}$ at the $\mathrm{VKa}$ peak position.

This discussion arose from a comment made by another analyst, who remarked that he "...was recently given another rutile standard...reputed to have $0.4 \% \mathrm{~V}$ by WDS analysis". My guess is that the analyst who originally performed the work did not correct for the overlap - it is very close to the $0.5 \%$ value, and one should in general be suspicious of trace quantities of $\mathrm{V}$ in $\mathrm{TiO}_{2}$.

In the absence of any more sophisticated interference correction software, you can make a correction for a simple overlap like this via:

\section{corrected V K-ratio = measured V K-ratio - (AK $x$ measured Ti K-ratio)}

where $A K$ is the apparent $V K$-ratio measured on pure $\mathrm{TiO}_{2}$ (here 0.0050). Thus, when analyzing pure $\mathrm{TiO}_{2}(\mathrm{Ti} K=1)$ the resulting $V$ concentration is zero, and when analyzing a phase containing no $\mathrm{Ti}(\mathrm{Ti} \mathrm{K}=0$ ) the correction is zero. I use this method to correct the K-ratio before it is passed to the ZAF program, but you could just as well make a small correction like this on the weight percent data. In practice, I find that this linear correction reduces the apparent $\mathrm{V}$ in pure $\mathrm{TiO}_{2}$ by an order of magnitude, i.e. from $0 . \times \%$ to $0.0 \times \%$, so especially several hundred $\mathrm{ppm} V$ in rutile should be viewed with skepticism. Remember that the requirement is that the $\mathrm{TiO}_{2}$ standard contain no vanadium. This is because you are assuming that the intensity measured at the $V$ Ka peak position is due entirely to the Ti Kb overlap.

So how do you demonstrate that the pure $\mathrm{TiO}_{2}$ does net contain any vanadium to begin with? Perform a wavelength scan on the $\mathrm{TiO}_{2}$ looking for the presence of the $V^{*} K b^{*}$ peak, since this is not overlapped. If you see this peak, then you know there is $V$ in the sample. The $V K b$ peak could as well be used for calibration in order to analyze the $\mathrm{TiO}_{2}$ without any need for interference correction, but the caveat is that the $V$ Kb peak intensity is roughly one tenth that of the $V$ Ka peak intensity, so counting statistics would be worse.

This highlights the importance of having access to pure element or oxide primary standards, because there is little uncertainty about their composition, and they can also be used as potential interference standards as well. These are then complemented by multi-element standard materials that might exhibit the peak overlap problems such as described above, and these secondary standards can be used to check the validity of your interference correction. One should be more conservative in accepting trace element data accompanying a new "standard" as well.

This Ti-V example is not a bad overlap, because the WDS resolution allows you to almost completely resolve the peaks to begin with. Consider $\mathrm{Pb}$ La on As Ka using LIF (the application is for sulfides or arsenides). This is a complete overlap with no real possible solution via this linear correction. The solution in a case like this is to use $\mathrm{As} \mathrm{Kb}$ and either $\mathrm{Pb} \mathrm{Lb}$ or $\mathrm{Pb} \mathrm{Ma}$ as your lines. As long as there are no absorption edges between the $\mathrm{Ka}$ and $\mathrm{Kb}$ lines, or $\mathrm{La}$ and $\mathrm{Lb}$ lines, the mass absorption coefficients are usable; one really has no other choice.

Paul K. Carpenter, California Institute of Technology 